\title{
Antimicrobial Metabolites from Halophilic Actinomycete Nocardiopsis sp. Al-H10-1 (KF384482) Isolated from Alang, Gulf of Khambhat, India
}

\author{
Nisha Trivedi ${ }^{1}$ and Jignasha Thumar ${ }^{2 *}$ \\ ${ }^{1}$ Department of Microbiology, School of Science, RK University, \\ Rajkot - 360020, Gujarat, India \\ ${ }^{2}$ Department of Microbiology, Government Science College, \\ Gandhinagar-382015, Gujarat, India \\ *Corresponding author
}

Keywords

Marine

actinomycetes; halophilic; $16 \mathrm{~S}$ rRNA gene sequencing; antimicrobial activity; spot inoculation method; optimization; OVAT method

Article Info

Accepted: 25 May 2021 Available Online: 10 June 2021
The overuse of antibiotics has resulted in the development of drug resistant, a major problem in disease curing processes i.e. development of drug resistance. The World Health Organization (WHO) released its first list of the most concerning pathogens for human health in 2017 which suggested that there are total 12 bacterial families which have developed multiple drug resistance and for which novel antibiotics are required immediately (WHO 2017). There is a requirement to explore some novel compounds to overcome this issue. Thus our study aimed at exploration of marine actinomycetes as a valuable resource for novel products with antimicrobial properties. The halophilic actinomycete Nocardiopsis sp. Al-H10-1 (KF384482) was isolated from saline water (20 m away from shore) of Alang coast (Gulf of Khambhat), Bhavnagar, Gujarat, India. The isolate Al-H10-1 was identified as Nocardiopsis sp. through rigorous morphological and cultural characteristics; the species was confirmed through $16 \mathrm{~s}$ rRNA phylogenetic analysis. The antimicrobial potential of Nocardiopsis sp. Al-H10$l$ was assessed against a range of Gram-positive and Gram-negative bacteria as well as three fungi, there it demonstrated antimicrobial activity against four Gram negative bacteria and one Gram positive bacteria. The isolate didn't show any antifungal activity. The results of optimization showed that the highest antimicrobial compound production was obtained in the presence of $0.5 \%$ starch, $10 \% \mathrm{NaCl}$ and $\mathrm{pH} 9$.

\section{Introduction}

The World Health Organization (WHO) released its first list of the most concerning pathogens for human health in 2017 which suggested that there are total 12 bacterial families who have developed multiple drug resistance and for which novel antibiotics are required immediately (WHO, 2017). The study by O'Neill suggests that every year approximately 7,00,000 people die because of drug resistant infections globally and if current trends continue, it will be increased by 10 million people per year by 2050 (O'Neill, 
2016). The multiple drug resistance can be developed in organisms by various types of mechanisms such as presence of antibiotic degrading enzymes, antibiotic altering enzymes, gene transfer processes like conjugation, transformation, transduction etc. So there is a need for searching naturally occurring novel antimicrobial compounds.

The natural products and microbial metabolites are used as the main bioactive scaffold for the development of the novel antibiotics instead of using the large synthetic combinatorial libraries of molecules to develop novel drugs (Challinor and Bode, 2015). During 1981 to 2014, total 1211 small molecule drugs were approved by the United States Food and Drug Administration (FDA), from which approximately $65 \%$ was accounted for chemicals produced in nature, or compounds based on them (Newman and Cragg, 2016). Actinomycetes have always been known as the biotechnologically and industrially important group of microorganisms as they are the great producers of many primary and secondary metabolites useful in a variety of fields including healthcare, agriculture, veterinary etc. (Barka et al., 2016). Various unexplored or underexplored ecosystems are the most promising source of novel actinomycetes able to produce large numbers of secondary metabolites (Dhakal et al., 2016; Subramani and Aalbersberg, 2013).

The Gulf of Khambhat is a coastal region situated in the Bhavnagar District of the Gujarat State of India. The uniqueness of this region is, its salinity and alkalinity which harbors various unidentified, unique haloalkaliphilic bacterial species which can produce valuable secondary metabolites. Alang is a small town located on the Gulf of Khambhat, considered as the world's largest graveyard of ships. It is well known for its ship breaking and recycling activities, because of which it is also known as the most polluted coastal region (Patel et al., 2018).

\section{Materials and Methods}

\section{Isolation}

The halophilic actinomycete, Nocardiopsis $s p$. Al-H10-1 was isolated from saline water (20 $\mathrm{m}$ away from shore) of Alang coast (Gulf of Khambhat), Bhavnagar, Gujarat, India. The water sample was filtered with whatman filter paper to remove fine soil particles and the filtrate was serially diluted. From the highest dilution containing tube, $0.1 \mathrm{ml}$ of the sample was spreaded separately on starch agar, starch casein agar and actinomycetes isolation agar (Hi-Media, India), containing 5- $10 \% \mathrm{w} / \mathrm{v}$ $\mathrm{NaCl}$. The plates were incubated at $30^{\circ} \mathrm{C}$; on sixth day a typical chalky white colony was picked up and re-streaked on starch agar slants supplemented with $10 \% \mathrm{w} / \mathrm{v} \mathrm{NaCl}$ to ensure the purity of the colony (Chakrabarti, 1998). The culture was maintained at $4^{\circ} \mathrm{C}$.

\section{Morphological and Biochemical Characteristics and Antibiotic Susceptibility Test}

Morphological, physiological and biochemical characteristics of the isolate were determined using standard methods. The morphological characteristics were determined by growing the isolate on a starch agar plate (with $10 \%$ $\mathrm{NaCl}$ ). After seven days of incubation, the colony characteristics were noted and Gram's staining was performed. Basic biochemical tests were performed for primary characterization of the isolate based on Bergey's manual of systematic Bacteriology Vol. IV (Garrity et al., 2004), which included including carbon sources utilization (Mannitol, Sucrose, Xylose, Glucose, Maltose, and Lactose), Methyl red test, VogesProskauer test, $\mathrm{H}_{2} \mathrm{~S}$ production, Catalase test, Indole production, Citrate utilization, Starch 
hydrolysis, Casein hydrolysis, Gelatin hydrolysis, Nitrate reduction, Urea hydrolysis, Ammonia production, and Triple sugar iron test. All media and test reagents were prepared as mentioned by Cappuccino and Sherman (2004). Antibiotic susceptibility and resistance pattern of the isolate were checked by disc diffusion method (Kumar et al., 2014). The test was conducted against total 13 antibiotics using Starch agar plates with $10 \% \mathrm{NaCl}$.

\section{Molecular Identification of Isolate by $16 \mathrm{~S}$ rRNA}

The molecular identification of the isolate AlH10-1 was carried out by $16 \mathrm{~S}$ rRNA gene sequencing. The 16SrRNA gene was amplified using universal primers $518 \mathrm{~F}$ 5'ccagcagccgcggtaatacg3' and 800R 5'taccagggtatctaatc3'. PCR products were purified and sequenced. The resultant sequences aligned within the NCBI database (National Centre for Biotechnology Information) using BLASTN. The phylogenetic tree was constructed using neighbor-joining with Kimura 2-state parameter and pairwise-deletion model analysis implemented in the program MEGA software version X (Kumar et al., 2018) and also evaluated further in a bootstrap analysis of 1,000 replicates. The 16SrRNA gene sequence of the isolate has been submitted to NCBI, GenBank, Maryland, USA.

\section{The Antimicrobial Potential}

Antimicrobial activity of Nocardiopsis sp. AlH10-1 was checked by spot inoculation method (Kumar et. al., 2010)using starch agar $(10 \% \mathrm{w} / \mathrm{v} \mathrm{NaCl})$. The spore suspension of the isolate was spotted on the medium and incubated at $28^{\circ} \mathrm{C}$ until sporulation. The test organisms, procured from MTCC, Chandigarh (ATCC equivalent), were used to check antimicrobial activity. These included Grampositive organisms: Bacillus subtilis,
Staphylococcus aureus, Micrococcus luteus, Staphylococcus epidermidis, Bacillus megaterium, Bacillus cereus; Gram-negative organisms: Pseudomonas aeruginosa, Escherichia coli, Enterobacter aerogenes, Serratia marcescens, Shigella flexneri, Salmonella enteric typhimurium, Proteus vulgaris, Klebsiella pneumoniae and fungi: Aspergillus niger, Fusarium oxysporum, Candida albicans. The test organisms were grown in nutrient broth at $37^{\circ} \mathrm{C}$ for 24 hours. The molten nutrient agar, with $1 \%$ activated test culture, was poured on the sporulated Nocardiopsis sp. Al-H10-1. After the incubation of 24 hours at $37{ }^{\circ} \mathrm{C}$, the zone of inhibition was measured for each test organism.

\section{Optimization of Growth Conditions for Antimicrobial Compound Production}

To achieve the highest production of antimicrobial compounds, effect of various growth conditions were studied by the one variable at a time (OVAT) method. To find out the optimized medium for the growth of the organism as well as for antimicrobial compounds production, the spore suspension of Nocardiopsis sp. Al-H10-1 was inoculated into various flask containing $20 \mathrm{ml}$ of starch broth, with respective starch concentration, $\mathrm{NaCl}$ concentrations and $\mathrm{pH}$ value separately. All the flasks were incubated at $30^{\circ} \mathrm{C}$ for $7-8$ days. Then the cell free filtrates of the culture were collected from all the media separately and were tested against the actively growing sensitive culture of Serratia marcescens by agar well diffusion method. The plates were incubated at $37^{\circ} \mathrm{C}$ for 24 hours followed by the measurement of zone of inhibition. The effect of starch as a carbon source on the growth and antimicrobial compound production was checked by using starch broth supplemented with various concentrations of starch $(0,0.5,1$ and $1.5 \%), 10 \% \mathrm{NaCl}$. The effect of the salt concentration on growth and the antimicrobial 
compound production was studied by using $1 \%$ starch broth provided with varying salt concentrations $(0,5,10$ and $15 \% \mathrm{w} / \mathrm{v} \mathrm{NaCl})$. The effect of $\mathrm{pH}$ on growth and antimicrobial compound production was studied by using $1 \%$ starch broth supplemented with $10 \%$ w/v $\mathrm{NaCl}$ and the range of $\mathrm{pH}(8,9,10$ and 11).

\section{Results and Discussion}

\section{Isolation and Morphology of the Organism}

Nocardiopsis sp. Al-H10-1, a halophilic actinomycete was isolated from the site $20 \mathrm{~m}$ away from Alang sea shore, Gulf of Khambhat, Western India. The isolate was characterized on the basis of its cell and colony morphology and Gram's reaction. The colonies were medium sized, irregular shaped, irregular, slightly raised, rough, and opaque with creamy white pigmentation. It was Grampositive, having a filamentous, long threadlike structure. It started sporulation on starch casein agar after 3 days of incubation with a fluffy mass of spores.

\section{Biochemical Characteristics and Antibiotic Susceptibility Test}

Nocardiopsis sp. Al-H10-1 is a Gram-positive, filamentous and sporulating organism. Various biochemical tests were performed for primary characterization of the isolate. Nocardiopsis sp. Al-H10-1 showed positive results for Voges-Proskauer test, Ammonia production, Catalase test, Citrate utilization, Triple sugar iron test, Starch hydrolysis, Casein hydrolysis and Gelatin hydrolysis while organism showed negative results for Methyl red test, Indole production, Urea hydrolysis test, Nitrate reduction test and $\mathrm{H}_{2} \mathrm{~S}$ production. The organism was grown in broth, supplemented with various carbohydrates like Mannitol, Sucrose, Xylose, Glucose, Maltose and Lactose to check its ability to utilize various carbohydrates and their fermentation pattern. Among the six provided carbohydrates, the organism was able to ferment glucose, sucrose, and maltose but not mannitol, xylose and lactose. The results of antibiotic susceptibility test showed that the isolate showed the highest sensitivity towards Rifampicin followed by Vancomycin, Ciprofloxacin, Gentamicin, Chloramphenicol, Streptomycin, Tetracycline, Kanamycin, Penicillin and Polymyxin-13 while the isolate was resistant towards Nalidixic acid, Ampicillin and Methicillin (Table 1).

\section{Molecular Identification of Nocardiopsis sp. Al-H10-1 through 16S rRNA Sequencing}

The 16S rRNA gene sequencing of the strain Nocardiopsis sp. Al-H10-1 showed the presence of $1457 \mathrm{bp}$ long 16S rRNA gene in the genomic sequence. The sequence was submitted to NCBI, GenBank, Maryland, USA with accession number (KF384482). The phylogenetic tree was constructed using neighbor-joining with Kimura 2-state parameter and pairwise-deletion model analysis implemented in the program MEGA software version X (Kumar et. al., 2018) and also evaluated further in a bootstrap analysis of 1,000 replicates (Figure 1). The molecular characterization through $16 \mathrm{~S}$ rRNA gene sequencing revealed the organism belonging to Nocardiopsis $s p$.

\section{The Antimicrobial Potential}

The antimicrobial potential of the isolate was checked against six Gram-positive, eight Gram-negative bacteria and three fungi. The isolate Nocardiopsis sp. Al-H10-1 inhibited the growth of the total five test organisms including four Gram negative bacteria Pseudomonas aeruginosa, Serratia marcescens, Enterobacter aerogenes, Klebsiella pneumoniae and one Gram positive bacterium Bacillus megaterium was inhibited (Figure 2). The highest inhibition was 
recorded against Serratia marcescens, however the isolate didn't show any inhibitory effect against any of the fungi tested for the same.

\section{Optimization of Growth Conditions for} Antimicrobial Compound Production

To achieve the highest production of antimicrobial compounds, effect of various growth conditions were studied. Optimization of various growth conditions showed that the highest antimicrobial compound production was obtained in the presence of $0.5 \%$ starch, $10 \% \mathrm{NaCl}$ and $\mathrm{pH} 9$ (Figure 3-5).

From 2007 to 2017 (the duration of 10 years), total 177 new species of rare actinomycetes were reported from the marine habitats representing 33 families from which 3 were novel families and 29 novel genera (Subramani and Sipkema, 2019). The study of Berdy, 2012 suggested that there are approximately 500,000 naturally occurring biological compounds, from which approximately 70,000 are microbially-derived with $29 \%$ is exclusively derived from actinomycetes. Marine actinomycetes are the prominent source of enzymes which can be produced on a large scale and can be used in various industries, such as proteases, cellulases, chitinases, amylases, xylanases, and other enzymes (Vaijayanthi et al., 2016).

They are also known as the most valuable source of naturally occurring novel antimicrobial compounds (Fiedler Forsyth et al., 1971; Hassanshahian and Mohamadian, 2011). So we focused on halophilic actinomycetes for the production of novel antimicrobial compounds in our research.

Nocardiopsis sp. Al-H10-1 was successfully isolated from the marine water sample from Alang sea shore using selective media supplemented with $10 \% \mathrm{NaCl}$. On the basis of morphological and molecular characterization, the isolate was identified as Gram-positive, filamentous, long thread-like structure containing Nocardiopsis sp. Al-H1O-1 able to inhibit the growth of four Gram negative bacteria including Pseudomonas aeruginosa, Serratia marcescens, Enterobacter aerogenes, Klebsiella pneumoniae and one Gram positive bacterium Bacillus megaterium. Das et al., (2018) isolated total 172 actinobacteria using soil samples collected from two microbiologically unexplored forest ecosystems, located in the Eastern Himalayan Biodiversity hotspot region i.e. Nameri National Park (NNP) and Panidehing Wildlife Sanctuary (PWS) by using the same isolation techniques used by us, they further screened 24 strong antimicrobial activity showing isolates using the same spot inoculation method.

Biochemical tests of Nocardiopsis sp. Al-H101 showed that the isolate is able to utilize various carbon sources like glucose, citrate, starch, sucrose and maltose; and nitrogen sources like casein and gelatin with production of ammonia. The organism is also able to produce various industrially important enzymes including amylase, proteases like caseinase and gelatinase. The isolate was able to produce catalase which indicates the organism is aerobic in nature.

The organism was able to ferment various carbohydrates like glucose, sucrose, and maltose. The negative results for $\mathrm{H}_{2} \mathrm{~S}$ production and nitrate reduction test indicated that the isolate is not able to utilize more complex protein contents and sulfur containing amino acids from the surrounding habitats. Antibiotic sensitivity/resistant profiling is widely used to study microbial diversity in combination with biochemical and molecular characterization of the isolates (Litzner et al., 2006). 
Table.1 Biochemical characteristics of Nocardiopsis sp. Al-H10-1

\begin{tabular}{|c|c|}
\hline Biochemical Test & Result \\
\hline Methyl red test & - \\
\hline Voges-Proskauer test & + \\
\hline Indole production & - \\
\hline Urease test & - \\
\hline Nitrate reduction test & - \\
\hline Ammonia production test & + \\
\hline Catalase test & + \\
\hline $\mathrm{H}_{2} \mathrm{~S}$ production test & - \\
\hline Citrate utilization test & + \\
\hline TSI & + \\
\hline Gelatin hydrolysis test & + \\
\hline Casein hydrolysis test & + \\
\hline Starch hydrolysis test & + \\
\hline Utilization of Carbohydrates & \\
\hline Mannitol & - \\
\hline Sucrose & + \\
\hline Xylose & - \\
\hline Glucose & + \\
\hline Maltose & + \\
\hline Lactose & - \\
\hline \multicolumn{2}{|c|}{ Antibiotic Susceptibility Test } \\
\hline Antibiotic (Concentration) & $\begin{array}{c}\text { Zone of Inhibition } \\
(\mathrm{mm})\end{array}$ \\
\hline Kanamycin $(30 \mu \mathrm{g})$ & $7(S)$ \\
\hline Streptomycin $(10 \mu \mathrm{g})$ & $21(\mathrm{~S})$ \\
\hline Nalidixic acid $(30 \mu \mathrm{g})$ & $0(\mathrm{R})$ \\
\hline Gentamicin $(10 \mu \mathrm{g})$ & $22(\mathrm{~S})$ \\
\hline Rifampicin $(5 \mu \mathrm{g})$ & $32(\mathrm{~S})$ \\
\hline Ciprofloxacin $(5 \mu \mathrm{g})$ & $23(\mathrm{~S})$ \\
\hline Ampicillin $(10 \mu \mathrm{g})$ & $0(\mathrm{R})$ \\
\hline Polymyxin-13 (300 units/disc) & $1(\mathrm{~S}$ \\
\hline Tetracycline $(30 \mu \mathrm{g})$ & $18(\mathrm{~S})$ \\
\hline Chloramphenicol $(30 \mu \mathrm{g})$ & $22(\mathrm{~S})$ \\
\hline Vancomycin $(30 \mu \mathrm{g})$ & $28(\mathrm{~S})$ \\
\hline Methicillin (5 $\mu \mathrm{g})$ & $0(\mathrm{R})$ \\
\hline Penicillin (10 units/disc) & $5(S)$ \\
\hline
\end{tabular}


Fig.1 Neighbor-joining tree of the strains and representative species of the genus Nocardiopsis and Streptomyces based on nearly complete $16 \mathrm{~S}$ rRNA gene sequences. Numbers at the nodes indicate the levels of bootstrap support based on 1000 resampled data sets. The scale bar indicates 0.01 substitution per nucleotide position

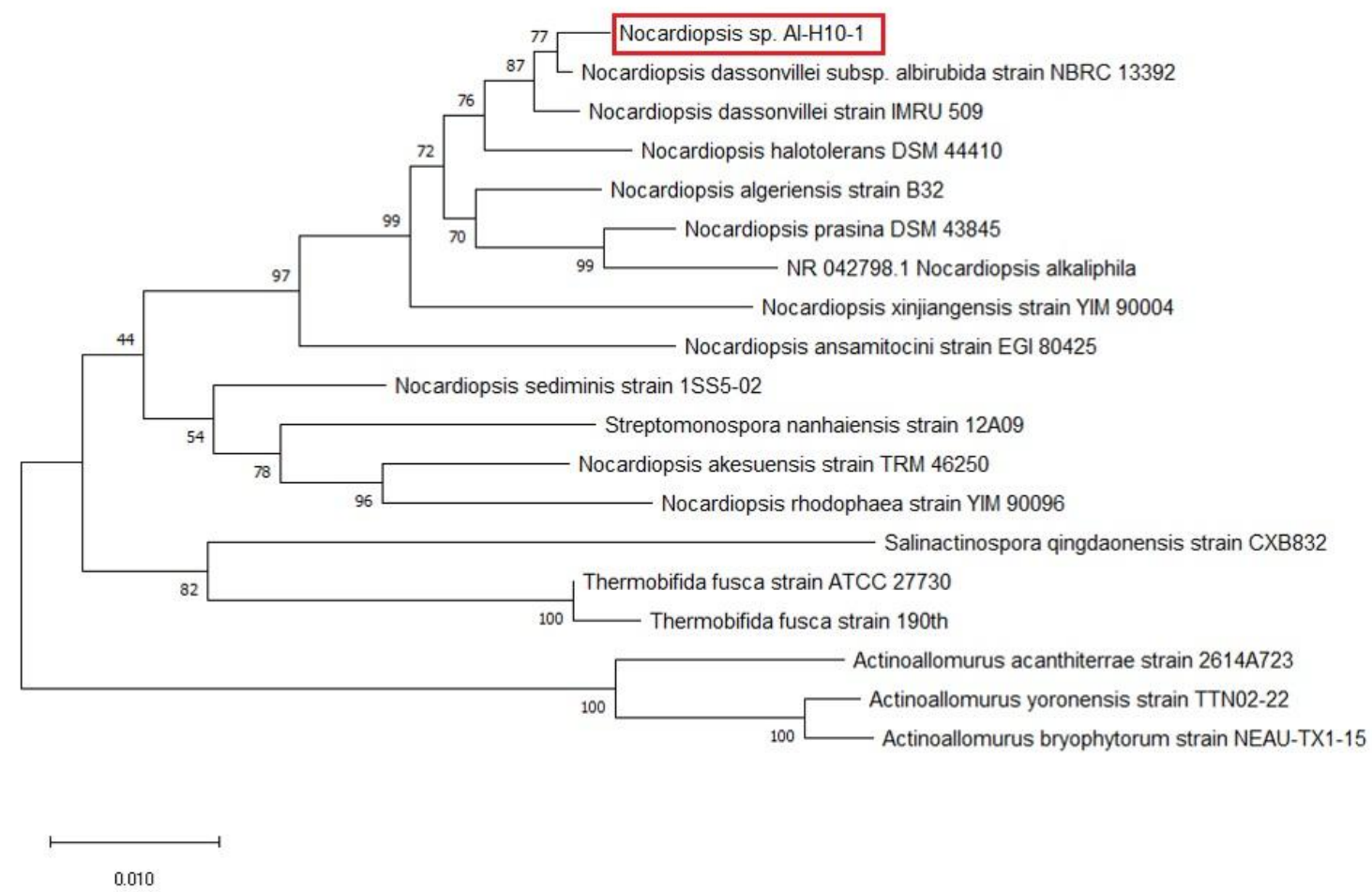

Fig.2 Antibacterial activity of Nocardiopsis sp. Al-H10-1 isolate against various test organisms

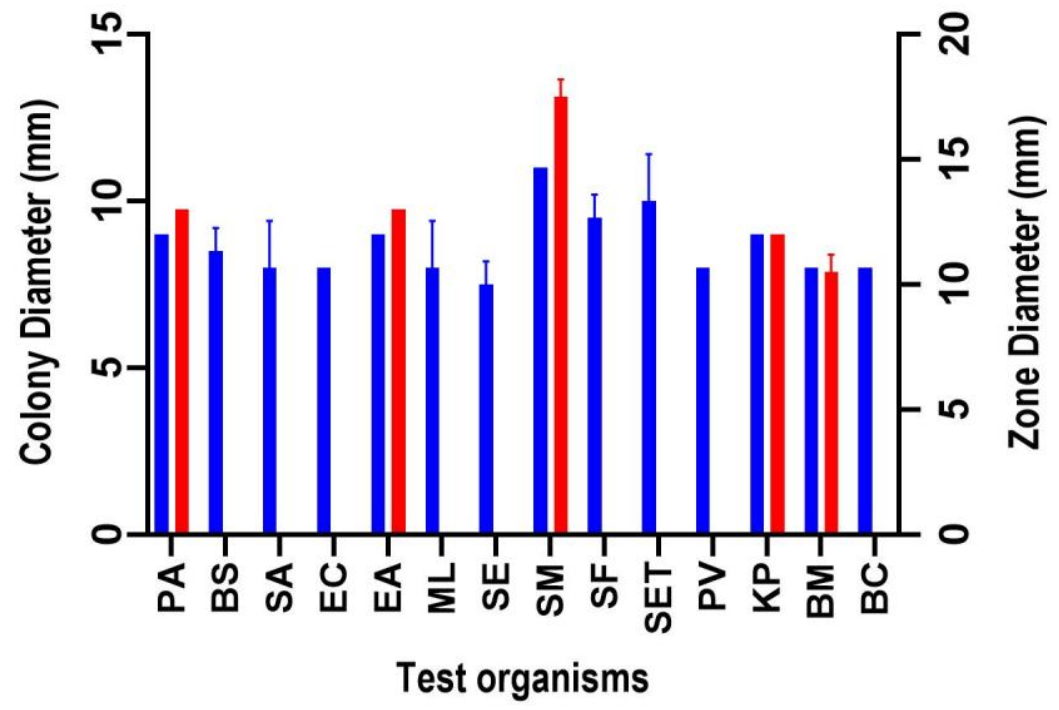

Colony Diameter ( $\mathrm{mm})$

Zone Diameter (mm) 
Fig.3 Effect of starch concentrations on antimicrobial compounds production by Nocardiopsis sp. Al-H10-1 against Serratia marcescens

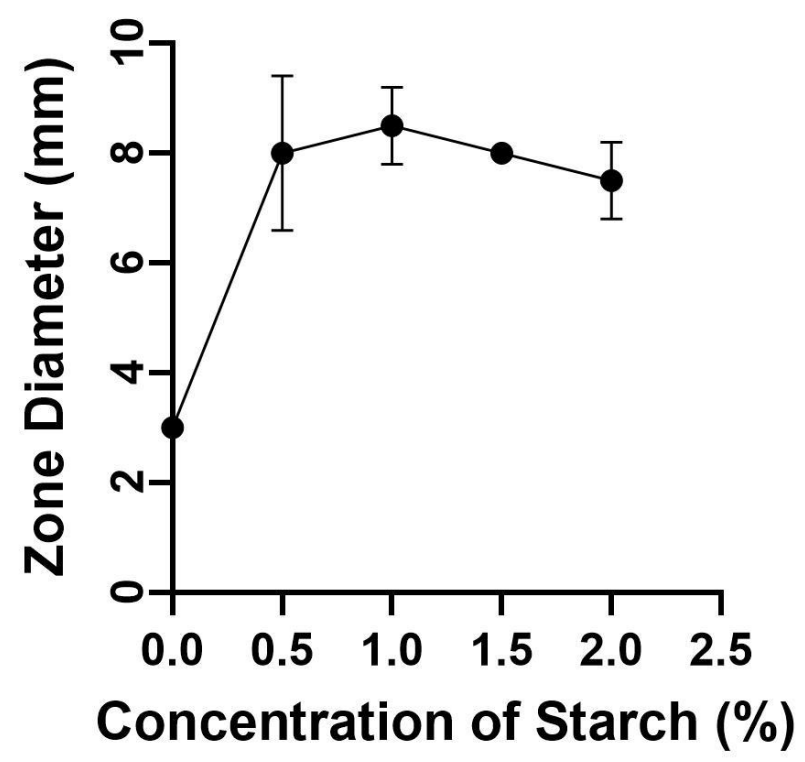

Fig.4 Effect of salt concentrations on antimicrobial compounds production by Nocardiopsis sp. Al-H10-1 against Serratia marcescens

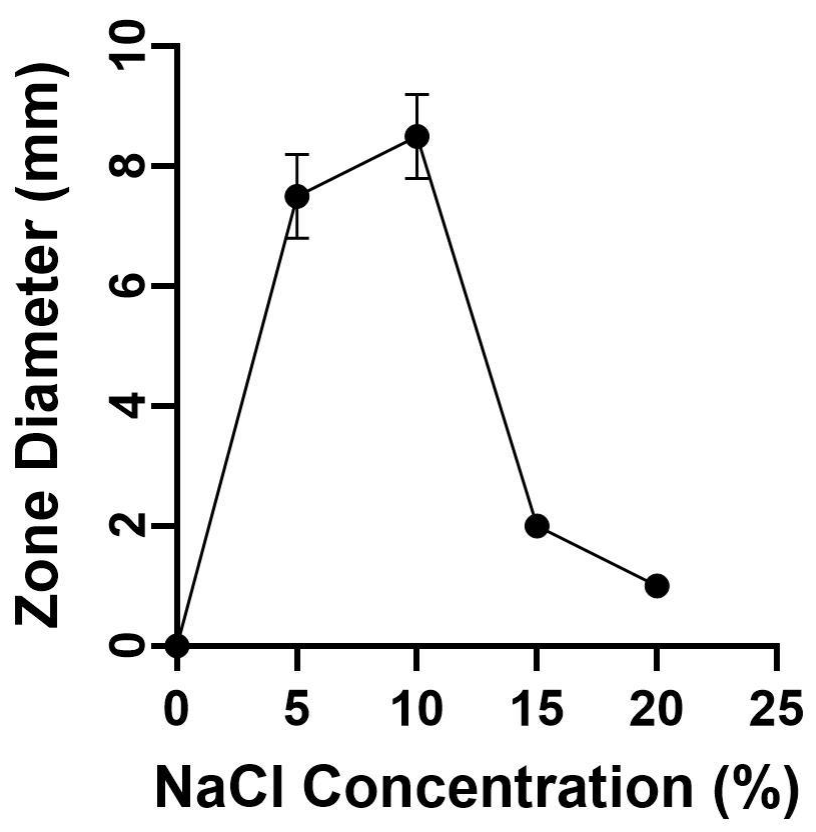


Fig.5 Effect of $\mathrm{pH}$ on antimicrobial compounds production by Nocardiopsis sp. Al-H10-1 against Serratia marcescens

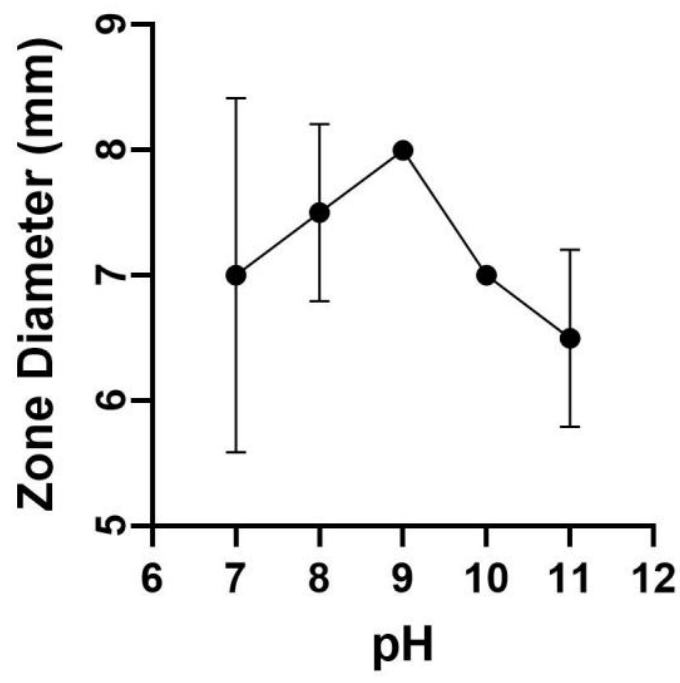

The antibiotic sensitivity/resistant profiling showed that the isolate is sensitive towards range of protein synthesis inhibiting antibiotics such as chloramphenicol, gentamycin, streptomycin and tetracycline. The highest sensitivity showed towards RNA synthesis inhibiting rifampicin followed by cell wall synthesis inhibiting vancomycin while the isolate showed resistance towards DNA synthesis inhibitor nalidixic acid and cell wall synthesis inhibitors ampicillin and methicillin. The phylogenetic analysis of the isolate showed that Nocardiopsis sp. Al-H10-1 showed the highest similarity with Nocardiopsis halotoleransDSM44410 strain NBRC100347 (98.63\%) and the lowest similarity with Actinoallomurus acanthiterrae strain 2614A723 (91.59\%).

The results of starch optimization showed that the isolate showed the highest production of antimicrobial compounds in the presence of $1 \% \mathrm{w} / \mathrm{v}$ starch concentration. The isolate was able to produce antimicrobial compounds even in the absence of starch.

The production of antimicrobial compounds was increased with increase in starch concentration up to $1 \% \mathrm{w} / \mathrm{v}$ starch concentration. Further increase in starch concentration reduced the production of antimicrobial compounds. Optimization of $\mathrm{NaCl}$ concentration for production of antimicrobial compounds showed that the highest amount of antimicrobial compounds was produced at $10 \% \mathrm{w} / \mathrm{v} \mathrm{NaCl}$ concentration.

The isolate didn't show any production of antimicrobial compounds at $0 \% \mathrm{w} / \mathrm{v} \mathrm{NaCl}$. The production of antimicrobial compounds was initiated at $5 \% \mathrm{w} / \mathrm{v} \mathrm{NaCl}$ concentration and increased up to $10 \% \mathrm{w} / \mathrm{v}$ of $\mathrm{NaCl}$ concentration. Further increase in $\mathrm{NaCl}$ concentration reduced the production of antimicrobial compounds.

The optimum $\mathrm{pH}$ for the production of antimicrobial compounds was $\mathrm{pH}$ 9. The isolate started production of antimicrobial compounds at $\mathrm{pH}$ 7. The production of antimicrobial compounds was increased with increase in $\mathrm{pH}$ up to $\mathrm{pH}$ 9. Further increase in $\mathrm{pH}$ showed reduction in the production of antimicrobial compounds. 


\section{Acknowledgement}

Financial Assistance from the Department of Biotechnology (New Delhi, India) is acknowledged.

\section{References}

Barka, E. A., Vatsa, P., Sanchez, L., GaveauVaillant, N., Jacquard, C., Klenk, H. P., Clément, C., Ouhdouch, Y. and van Wezel, G. P. 2016. Taxonomy, physiology, and natural products of Actinobacteria. Microbiol. Mol. Biol. Rev., 80, 1-43.

Berdy, J. 2012. Thoughts and facts about antibiotics: Where we are now and where we are heading. J. Antibiot., 65, 385-395.

Cappuccino, J. G. and Sherman, N. 2004. Microbiology, a laboratory manual, 6th edn. Pearson education, Singapore

Chakrabarti, T. 1998. Actinomycetes Isolation, Screening, Identification, and Gene Cloning in Streptomyces Laboratory manual.

Challinor, V. L. and Bode, H. B. 2015. Bioactive natural products from novel microbial sources. Ann. N. Y. Acad. Sci., 1354, 82-97.

Das, R., Romi, W., Das, R., Sharma, H. K. and Thakur, D. 2018. Antimicrobial potentiality of actinobacteria isolated from two microbiologically unexplored forest ecosystems of Northeast India. BMC Microbiology, 18(1). https://doi.org/10.1186/s12866018-1215-7

Dhakal, D., Pokhrel, A. R., Shrestha, B. and Sohng, J. K. 2017. Marine rare actinobacteria:

Isolation, characterization, and strategies for harnessing bioactive compounds. Front. Microbiol., 8, 1106.

Fiedler Forsyth, M. P., Shindler, D. B., Gochnauer, M. B. and Kushner, D. J.
1971. Salt tolerance of intertidal marine bacteria. Can J Microbiol 17:825-828.

Garrity, G. M., Bell, J. A. and Lilburn, T. G. 2004. Taxonomic outline of the prokaryotes. Bergey's manual of systematic bacteriology. New York.

Hassanshahian, M. and Mohamadian, J. 2011. Isolation and characterization of Halobacterium in Iran. Jundishapur J Microbiol 4:S59-S65.

Kumar, N., Singh, R. K., Mishra, S. K., Singh, A. K. and Pachouri, U. C. 2010. Isolation and screening of soil Actinomycetes as source of antibiotics active against bacteria. Int. J. Microbiol. Res., Volume 2, Issue 2, pp-12-16, ISSN: 0975-5276.

Kumar, P. S., Duraipandiyan, V. and Ignacimuthu, S. 2014. Isolation, screening and partial purification of antimicrobial antibiotics from soil Streptomyces sp. SCA 7. Kaohsiung J Med Sci., 30 (9): 435-446.

Kumar, S., Stecher, G., Li, M., Knyaz, C. and Tamura, K. 2018. MEGA X: Molecular Evolutionary Genetics Analysis across Computing Platforms. Mol. Biol. Evol., 35, 1547-1549.

Litzner, B. R., Caton, T. M. and Schneegurt, M. A. 2006. Carbon substrate utilization, antibiotic sensitivity, and numerical taxonomy of bacterial isolates from the Great Salt Plains of Oklahoma. Arch Microbiol 185:286296

Newman, D. J. and Cragg, G. M. 2016. Natural Products as sources of new drugs from 1981 to 2014. J. Nat. Prod., 79, 629-661.

O’Neill, J. 2016. Tackling drug-resistant infections globally: final report and recommendations [Internet]. London (UK): The review on antimicrobial resistance; [cited 2018 May 8]. Available from https://amr- 
review.org/sites/default/files/160525_F inal\%20paper_with\%20cover.pdf

Patel, A. B., Mahala, K., Jain, K. and Madamwar, D. 2018. Development of mixed bacterial cultures DAK11 capable for degrading mixture of polycyclic aromatic hydrocarbons (PAHs). Bioresour. Technol., 288296.

Subramani, R. and Aalbersberg, W. 2013. Culturable rare actinomycetes: Diversity, isolation and marine natural product discovery. Appl. Microbiol. Biotechnol., 97, 9291-9321.

Subramani, R. and Sipkema, D. 2019. Marine Rare Actinomycetes: A Promising Source of Structurally Diverse and Unique Novel Natural Products. Mar.
Drugs, $\quad 17, \quad 249 . \quad$ doi: https://www.mdpi.com/16603397/17/5/249

Vaijayanthi, G., Vijayakumar, R. and Dhanasekaran, D. 2016. 'Actinobacteria - A Biofactory of Novel Enzymes', in ActinobacteriaBasics and Biotechnological Applications, eds D. Dhanasekaran, and Y. Jiang, (Norderstedt: BoD), 88111.

WHO (2017). Global priority list of antibioticresistant bacteria to guide research, discovery, and development of new antibiotics [Internet]. Geneva (Switzerland): World Health Organization.

\section{How to cite this article:}

Nisha Trivedi and Jignasha Thumar. 2021. Antimicrobial Metabolites from Halophilic Actinomycete Nocardiopsis sp. Al-H10-1 (KF384482) Isolated from Alang, Gulf of Khambhat, India. Int.J.Curr.Microbiol.App.Sci. 10(06): 729-739. doi: https://doi.org/10.20546/ijcmas.2021.1006.080 